\title{
Détermination d'un Nouveau Système de Critères de Transfert de L'état Vers les Collectivités Territoriales au Maroc
}

\author{
Driss Effina $^{1}$ and Othmane Fahim ${ }^{2}$ \\ ${ }^{1}$ Economic \& Finance Department, INSEA, Rabat, Morocco \\ ${ }^{2}$ Economic Department, University Mohammed V, Rabat, Morocco
}

\begin{abstract}
Résumé : Les inégalités territoriales renvoient généralement à une situation de déséquilibre entre les territoires. Il s'agit d'un déséquilibre économique, social et de bien-être entre les populations des différents territoires du pays. Les écarts entre les populations et les territoires sont quantifiables à travers plusieurs indicateurs socio-économiques et culturels. Le phénomène des inégalités territoriales au Maroc traduit une situation de déficit structurel. Des retards sont observés en matière de développement social et humain dans les différentes régions. Pour remédier à ces carences, le modèle de solidarité territoriale en vigueur est construit autour de plusieurs mécanismes de financement (BGE, ressources des CT, fonds spécialisés). Ces mécanismes sont loin de pouvoir combler les déficits et répondre aux besoins exprimés par les collectivités territoriales. De plus, ce modèle connait l'intervention de plusieurs acteurs (Etat, CT...) sans un cadre de gouvernance bien défini. Ce papier propose un nouveau système de critères et d'orientations pour améliorer l'efficacité du système de solidarité en vigueur afin d'accélérer la résorption des déficits sociaux, de lutter contre les déficits anciens et de créer une équité en matière de répartition de ressources financières transférées du central vers les territoires.
\end{abstract}

Mots-clés : Transferts de l’Etat, Collectivités territoriales, Péréquation, Solidarité territoriale

\section{INTRODUCTION}

Le transfert financier de l'Etat vers les collectivités territoriales (CT) constitue un mécanisme universel de la solidarité territoriale. Le Maroc n'échappe pas à cette pratique répandue dans de nombreux pays. Le système de solidarité territoriale marocain repose sur la péréquation verticale consistant en un transfert de l'Etat vers les CT des dotations budgétaires sous forme de part des impôts et taxes de TVA, d'IS, d'IR et de TCA.

La répartition de ces dotations obéit à des critères ne reflétant pas les besoins réels des collectivités. Cette répartition ne s'accorde pas parfaitement avec le niveau de développement socio-économique des territoires. Ainsi, les collectivités se trouvent en difficulté de disposer des moyens nécessaires pour lutter contre les inégalités territoriales se manifestant au sein des sous-territoires. Ce papier a pour objet de proposer les mécanismes d'opérationnalisation de la solidarité territoriale. Il s'agit d'éclater les mécanismes à même de renforcer la solidarité territoriale et consolider les efforts consentis par l'Etat et les collectivités territoriales dans la lutte contre les inégalités territoriales au Maroc.

Dans ce cadre, cet article se propose d'établir un nouveau système de critères de transferts de l'Etat vers les collectivités territoriales. Ce nouveau système est axé sur la place centrale du citoyen dans le système de solidarité territoriale. Il se réfère ainsi à l'importance que doit jouer la région dans la lutte contre les inégalités territoriales. 
Ce système tient aussi compte du dualisme urbain/rural entre les territoires. De plus, le système proposé est un système orientant les investissements publics, toutes formes confondues, vers les territoires nécessiteux. Il permet de drainer la composante sociale des investissements publics vers toutes les régions en tenant compte des déficits socio-économiques enregistrés dans les territoires. Ce système donne ainsi l'occasion de bien répartir ces investissements au sein du territoire régional conformément aux orientations de la planification et de l'aménagement du territoire régional. Le système proposé est de nature à renforcer l'équité entre les citoyens, quels que soient leurs lieux de résidence. Il est également d'essence à consolider la solidarité entre les territoires. Ainsi, il permet de soutenir l'efficacité et l'efficience de l'action publique de développement et de lutte contre les inégalités territoriales.

Le présent papier s'articule, comme suit: nous présenterons l'intercommunalité comme mécanisme institutionnel de la solidarité territoriale, suivi des différents critères d'octroi des ressources des fonds d'intervention pour la lutte contre les inégalités territoriales. Puis, nous discuterons d'un modèle alternatif de solidarité territoriale et de l'introduction des leviers de solidarité infrarégionale. Et enfin, nous examinerons le nouveau modèle proposé des transferts de l'Etat vers les collectivités territoriales, en deux niveaux.

\section{LE MECANISME INSTITUTIONNEL DE LA SOLIDARITE TERRITORIALE : L'INTERCOMMUNALITE}

L'intercommunalité se présente comme un regroupement de plusieurs collectivités territoriales de même ou de différente nature dans un cadre de coopération et sous forme d'un établissement public pour réaliser des projets communs ou gérer des services d'intérêt général pour le groupement (loi organique 111.14, art. 155). Ladite coopération entre les collectivités territoriales se fonde sur la libre volonté de celles-ci d'élaborer et de réaliser, des projets ou des services d'intérêt général au sein des périmètres de solidarité. Cette démarche permet de mutualiser des ressources communes et de partager les profits et les bénéfices à moindre coût. Elle permet également aux collectivités de surmonter leurs problèmes liés notamment aux déficits socio-économiques enregistrés (inégalités territoriales). Ainsi, c'est l'occasion d'entreprendre et de mener à bien une politique d'aménagement et de développement solidaire de leurs territoires.

Le principe d'intercommunalité permet aux collectivités contractantes de rattraper le déficit enregistré en matière d'infrastructures et d'équipements publics. Il joue un rôle redistributif important et permet aux collectivités regroupées d'améliorer leurs capacités financières et d'optimiser leurs charges. Aussi, cet instrument permet de distribuer les charges inhérentes au développement et à la gestion des projets entre les sous-territoires regroupés (Figure 1).

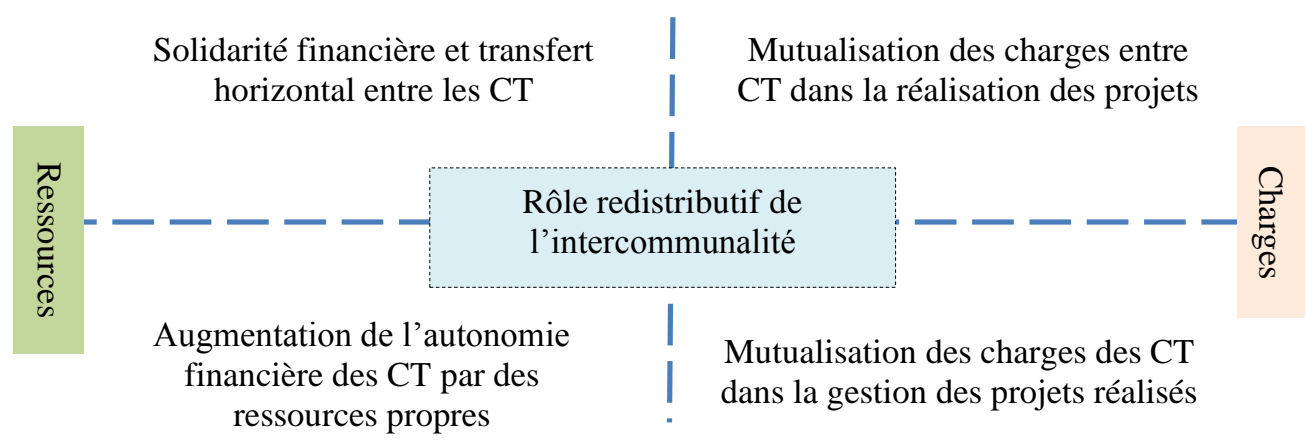

Figure 1. Rôle redistributif de l'intercommunalité 
Malgré les avantages de l'intercommunalité, cette dernière relève plus de l'exception que de la règle. Les cas de la coopération entre collectivités territoriales sont plutôt rares, et le nombre de groupements ne dépasse pas 111 pour l'ensemble du pays. Cette performance est au-dessous des possibilités offertes par cette forme institutionnelle et loin de faire face aux déficits cumulés en matière de services publics et des infrastructures. Par ailleurs, sa pratique actuelle se limite à la gestion des services publics communaux (transport public, décharges publiques, gestion des parcs naturels), ce qui le prive de jouer le rôle de mécanisme de solidarité territoriale. A ce jour, l'intercommunalité reste encore une pratique embryonnaire. Sur les 1503 communes, 75 provinces et 12 régions constituant le paysage territorial du Royaume, un énorme potentiel de coopération reste à explorer.

L'intercommunalité aussi se limite souvent au niveau communal sans franchir une coopération régionale ou interprovinciale. Plusieurs champs peuvent être investis par cette forme de coopération institutionnelle (loi organique 111.14, art. 154). La coopération intercommunale doit concerner davantage des champs d'action promoteurs de la solidarité territoriale. Les champs liés à la lutte contre les inégalités territoriales sont énumérés non exhaustivement (tableau 1) :

TABLE I. INTERCOMMUNALITÉ \& CHAMPS DE LUTTE CONTRE LES INÉGALITÉS TERRITORIALES

\begin{tabular}{|c|c|c|c|c|}
\hline Domaine & Projets \& Actions & CT concernées & Avantages & Modalités \\
\hline $\begin{array}{l}\text { Développement } \\
\text { économique }\end{array}$ & $\begin{array}{l}\text { Création de zones } \\
\text { d'activités } \\
\text { économiques, } \\
\text { touristiques, } \\
\text { industrielles... }\end{array}$ & $\begin{array}{l}\text { Région \& } \\
\text { communes }\end{array}$ & $\begin{array}{l}\text { Mutualisation des } \\
\text { charges de la } \\
\text { réalisation } \\
\text { Augmentation des } \\
\text { ressources propres }\end{array}$ & $\begin{array}{c}\text { Création de } \\
\text { groupement avec } \\
\text { des compétences } \\
\text { élargies }\end{array}$ \\
\hline $\begin{array}{l}\text { Développement } \\
\text { social }\end{array}$ & $\begin{array}{c}\text { Réalisation des } \\
\text { infrastructures et } \\
\text { équipements de base } \\
\text { (routes, } \\
\text { assainissement, } \\
\text { écoles, centres } \\
\text { culturels, sportifs...) }\end{array}$ & $\begin{array}{l}\text { Province \& } \\
\text { communes }\end{array}$ & $\begin{array}{l}\text { Mutualisation des } \\
\text { charges de la } \\
\text { réalisation }\end{array}$ & \\
\hline
\end{tabular}

Source : ministère de l'Intérieur, réalisé par nos soins

L'activation de l'intercommunalité comme mécanisme institutionnel de la solidarité territoriale permet de mener une action solidaire au profit des territoires. Afin de s'inscrire dans cette dynamique de solidarité et de coopération, plusieurs possibilités sont à explorer :

- Sensibiliser et motiver les collectivités sur l'importance de se regrouper. Créer un groupement solidaire permet des avantages : la mutualisation des efforts et des moyens pour répondre aux attentes des populations en termes de réduction des déficits sociaux relatifs aux équipements de base et aux infrastructures; l'intervention sur des territoires voisins permettant de satisfaire les attentes des populations et créer une complémentarité territoriale ; la disposition d'un cadre de négociation avec l'Etat pour drainer plus de ressources financières ; la spécialisation du groupement dans la réalisation des projets de différentes natures ce qui lui permet une intervention ciblée permettant de couvrir des domaines spécifiques qui relèvent de la solidarité territoriale; l'autonomie offerte par le statut du groupement et sa disposition des organes de gestions autonomes et des moyens propres est une opportunité pour accélérer les actions de la réduction des inégalités territoriales et l'intervention dans 
des territoires bien identifiés; à travers la sensibilisation des élus de l'importance d'adhérer à ce mécanisme, une motivation et une mobilisation peuvent avoir lieu pour créer davantage des groupements permettant de réaliser des projets de grande envergure ; renforcer la capacité des élus pour assurer la gestion des projets de développement.

- Axer l'intercommunalité sur une coopération de projet. La coopération entre les collectivités doit s'orienter vers une coopération de projet. Cette catégorie de coopération vise la réalisation des projets socio-économiques ayant pour objectif de doter les territoires des infrastructures de base, la réalisation des projets de développement social et l'amélioration du cadre de vie des populations. Cette forme de coopération ouvre des perspectives parce qu'elle peut concerner l'ensemble des communes d'une province, mais faire aussi l'objet d'une coopération entre communes frontalières appartenant à des provinces différentes. Elle a la particularité de se projeter dans l'avenir pour mieux maîtriser, en les organisant, les évolutions inévitables d'un monde en mouvement. Dans ce sens, il est opportun d'assoir la coopération de projet pour mener des projets d'aménagement des espaces notamment sensibles et l'organisation des activités sur les territoires concernés. Aussi, cette coopération doit se pencher sur des projets créateurs de richesse et d'emploi pour la population des territoires objets de la coopération.

- Choisir le périmètre de coopération pour répondre aux exigences de la réduction des inégalités territoriales. Le choix du périmètre de coopération s'avère important pour garantir la réussite de toute coopération entre les collectivités. En fait, ce choix de périmètre doit répondre à plusieurs critères relatifs à la situation géographique, sociale et économique ainsi que celle des inégalités au sein des collectivités objets de la coopération. En fait, la coopération permet de mutualiser les moyens pour faire face à des situations de déficits existantes au niveau des territoires. Ceci dit, il est nécessaire de faire une évaluation de la situation des inégalités territoriales, des potentialités de développement, des capacités de mobilisation des moyens et les perspectives de la réussite de la coopération. Une fois le périmètre déterminé, une formalisation de la coopération doit être faite à travers la création du groupement et l'élection de ses organes de gestion et sa dotation des moyens financiers et des apports de chaque collectivités. Ainsi, le groupement peut bénéficier des transferts de l'Etat pour soutenir la réalisation des projets définis. L'intercommunalité constitue alors un mécanisme pratique pour concrétiser la solidarité territoriale. A travers son activation par le biais des actions proposées, il est possible de renforcer le recours des collectivités à cette option importante pour mener une action solidaire contre les inégalités territoriales. Il est important de rappeler que ce mécanisme est très répandu dans différents pays du monde, notamment la France. L'intercommunalité en France constitue un outil indirect de la solidarité horizontale via la mutualisation des ressources pour la réalisation des projets de développement.

\section{CRITÈRES D'OCTROI DES RESSOURCES DES FONDS D'INTERVENTION POUR LA LUTTE CONTRE LES INÉGALITÉS TERRITORIALES : MODÈLE DE FSHIU}

Plusieurs fonds sont institués pour financer les opérations et les actions de lutte contre les inégalités territoriales. Il s'agit particulièrement du Fonds de financement de l'INDH, du Fonds Solidarité Habitat et Intégration Urbaine (FSHIU), (art. 28, loi de finances 2012), du Fonds pour le financement de programme de lutte contre les disparités territoriales en milieu rural, le Fonds de Développement Rural (FDR), le Fonds de Mise à Niveau social et le Fonds de Solidarité interrégionale. 
L'ensemble de ces fonds interviennent pour financer les actions de lutte contre les inégalités territoriales et de créer un équilibre social entre les différents territoires.

Néanmoins, la répartition des dotations de ces fonds risque de ne pas cibler les territoires dépourvus, ainsi, de ne pas réaliser les actions souhaitées dans une logique d'efficience et d'efficacité. Dans ce sens, il est important de revoir les critères de distribution des ressources de ces fonds pour mener à bien les actions de lutte contre les disparités territoriales. Afin d'illustrer le nouveau mode de financement des actions de lutte contre les inégalités territoriales, une grille de critère est à adopter pour cibler les territoires dépourvus. À titre d'exemple, le FSHIU est alimenté par la taxe spéciale sur le ciment (TSC) au taux de $0,05 \mathrm{dhs} / \mathrm{kg}$. Cette taxe a connu deux hausses pour passer à $0,10 \mathrm{dhs} / \mathrm{kg}$ en 2004 et $0,15 \mathrm{dhs} / \mathrm{kg}$ en 2012. Aussi, la loi de finances de 2013 a institué de nouvelles ressources pour le FSHIU à savoir la taxe spéciale sur le fer à béton, et $30 \%$ de la taxe spéciale sur le sable. Le FSHIU dépend largement des recettes de la TSC. Cette taxe constitue plus de $90 \%$ des recettes du fonds. Les différentes taxes alimentant le FSHIU ont permis d'augmenter ses ressources pour passer de 400 millions en 2002 à plus de 2,5 milliards de dhs en 2018 (tableau 2).

TABLE II. RECETTES FSHIU PAR TAXE (2010-2018)

\begin{tabular}{|c|c|c|c|c|c|c|c|c|c|}
\hline & & & & & & & \multicolumn{3}{|c|}{ En millions de dirham } \\
\hline & 2010 & 2011 & 2012 & 2013 & 2014 & 2015 & 2016 & 2017 & 2018 \\
\hline TSC & 1333,7 & 1514,1 & 1852,6 & 1735,1 & 2109 & 2137,7 & 2122,6 & 2068,4 & \\
\hline TS sable & & & & 147,3 & 147,1 & 174,3 & 149,5 & 256,1 & \\
\hline TS RB & & & & 87,5 & 60,1 & 58,1 & 40,9 & 74,5 & \\
\hline Total recettes & 1668,4 & 2092,6 & 1925,7 & 2464,5 & 2161 & 2397 & 2313 & 2399 & 2537,5 \\
\hline
\end{tabular}

Durant la période 2002-2018, étant donné son rôle de financement des opérations de lutte contre l'habitat insalubre, le FSHIU a permis le financement de plusieurs programmes d'habitat. Cet effort de financement des programmes de lutte contre l'habitat insalubre a connu une inflexion depuis 2012, en renforçant les dépenses destinées au financement des opérations de mise à niveau urbaine et celles de la politique de la ville.

TABLE III. DÉPENSES FSHIU PAR PROGRAMME 2018

\begin{tabular}{lcc}
\hline Programme & Total (MDH) & Part \% \\
Restructuration des QHNR & 11545,41 & $32 \%$ \\
Politique de la ville (PV) & 6527,07 & $18 \%$ \\
Programme Ville Sans Bidonvilles (VSB) & 8872,90 & $25 \%$ \\
Provinces du SUD (PS) & 2728,99 & $8 \%$ \\
Réhabilitation des tissus anciens et habitat menaçant ruine (HMR) & 2269,86 & $6 \%$ \\
Autres & 3512,67 & $10 \%$ \\
Total des dépenses par programme & 35740,41 & $\mathbf{1 0 0 \%}$ \\
\hline
\end{tabular}

Source : MEF, Département de l'Habitat (2019)

L'intervention dans les quartiers d'habitat non réglementaire (QHNR) et les opérations réalisées dans le cadre de la politique de la ville ont représenté la moitié des dépenses du FSHIU. Cette situation montre une régression des dépenses liées à la lutte contre les bidonvilles et l'importance des interventions liées à l'amélioration des conditions de vie dans le cadre bâti. 
Effina et al.: Détermination d'un nouveau système de critères de transfert de l'Etat vers les collectivités territoriales...

TABLE IV. RÉPARTITION DES SUBVENTIONS DU FSIU ET DU BGE PAR RÉGION (MDH) POUR LE PROGRAMME QHNR

\begin{tabular}{cccccccc}
\multirow{2}{*}{ Région } & \multicolumn{3}{c}{ Coût (MDH) } & \multicolumn{4}{c}{$\begin{array}{c}\text { Subvention totale } \\
\text { (FSHIU+BGE) }\end{array}$} \\
\cline { 2 - 8 } & FSHIU & BGE & Total & FSHIU & BGE & Total & \% \\
\hline Tanger Tétouan Al Hoceima & 1666 & 1219 & 2885 & 1187 & 560 & 1747 & 17 \\
\hline Oriental & 1244 & 498 & 1742 & 1150 & 488 & 1638 & 16 \\
\hline Fès Meknès & 1713 & 281 & 1994 & 1280 & 160 & 1440 & 14 \\
\hline Marrakech Safi & 3146 & 206 & 3351 & 1350 & 88 & 1437 & 14 \\
\hline Casablanca Settat & 2286 & 69 & 2355 & 1313 & 67 & 1380 & 13 \\
\hline Rabat Salé Kenitra & 1101 & 1687 & 2789 & 593 & 147 & 740 & 7 \\
\hline Beni Mellal Khénifra & 1009 & 51 & 1059 & 711 & 49 & 760 & 7 \\
\hline Souss Massa & 838 & 251 & 1089 & 410 & 99 & 509 & 5 \\
\hline Draa Tafilalt & 489 & 104 & 593 & 374 & 60 & 434 & 4 \\
\hline Guelmim Oued Noun & 244 & 248 & 493 & 242 & 202 & 444 & 4 \\
\hline Total & $\mathbf{1 3 7 3 6}$ & $\mathbf{4 6 1 5}$ & $\mathbf{1 8 3 5 0}$ & $\mathbf{8 6 0 8}$ & $\mathbf{1 9 2 1}$ & $\mathbf{1 0 5 2 9}$ & $\mathbf{1 0 0}$ \\
\hline \multicolumn{2}{c}{ Source : Année 2017, Département de l'Habitat et MEF } & & &
\end{tabular}

Les chiffres du tableau 4 montrent que ce sont les régions de Tanger-Tétouan-Al Hoceima, l'Oriental, Marrakech Safi, Fès-Meknès et Casablanca-Settat qui totalisent la plus grande part des subventions accordées étant donnée l'importance de ce type d'habitat dans ces régions. Le FSHIU connait des carences en matière de gestion, ce qui limite son efficacité dans la réalisation des missions pour lesquelles il a été destiné à l'origine (Cour des Comptes, 2015).

Le $1^{\text {er }}$ constat est l'importance des dépenses liées aux opérations de mise à niveau urbaine et la faiblesse du financement des programmes de l'habitat insalubre et des programmes de l'habitat social, qui constituent les principales missions du Fonds. Le $2^{\mathrm{e}}$ constat est le fait que le FSHIU finance plus de $67 \%$ des projets conventionnés et parfois la totalité des projets à cause de la faible contribution des autres partenaires publics et le non-respect de leurs engagements, en plus de la non-maîtrise des montages technico-financiers des opérations. Le $3^{\mathrm{e}}$ constat réside dans la hausse excessive des engagements (25 milliards de dirhams à la fin de l'année 2013) en l'absence des crédits permettant de les couvrir. Cette situation dénote d'un problème de gestion de ce fonds.

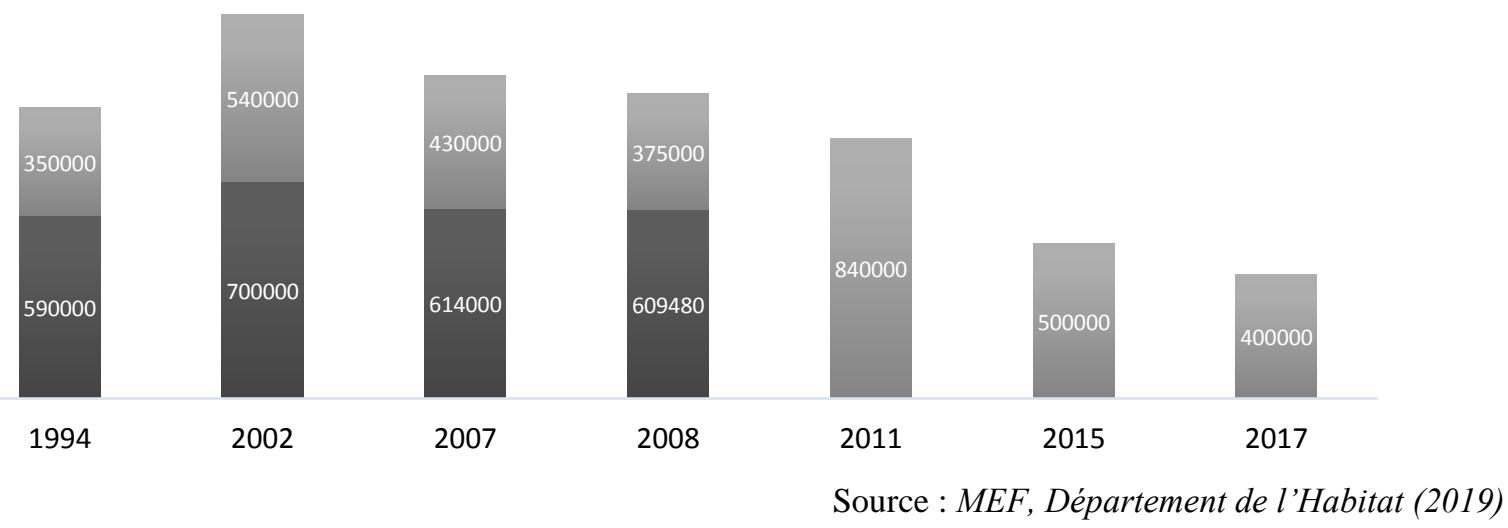

Figure 2. Evolution du déficit en logement 
Le déficit en logements s'est inscrit, en net repli, passant de 1,24 million d'unités en 2002 à 400.000 unités en 2017, soit une baisse de $67 \%$ (Figure 2). Un tel résultat est le corolaire des efforts déployés en matière de lutte contre l'habitat insalubre, de résorption des bidonvilles, de restructuration de l'habitat non règlementaire et de promotion du logement social et à faible valeur immobilière.

L'effort consenti pour la diminution du déficit en logement est fait principalement dans le cadre du programme VSB qui a permis durant la période 2004-2018 de reloger 278.000 ménages et de déclarer 59 villes sans bidonvilles. Aussi, les opérations de mise à niveau urbaine (MANU) ont permis d'améliorer le cadre de vie de 895.000 ménages sur la période 2006-2017. En fait, cette avancée est le fruit de l'effort de financement de FSHIU des différents programmes. Néanmoins, cet effort de financement a bénéficié pour certaines régions, plus que d'autres. En effet, 14\% des déblocages des crédits FSH sur la période 2002-2012 ont été attribués à la région de l'Oriental au moment où des régions qui souffraient d'un déficit très important comme Beni Mellal Khénifra, et Fès-Meknès n'ont bénéficié, respectivement, que de $3 \%$ et de $6 \%$.

La répartition de l'effort de financement de FSHIU doit prendre en considération de nouveaux critères pour permettre une répartition équitable au service de lutte contre les disparités territoriales en matière d'habitat, et partant sur le plan social. Plusieurs composantes s'affichent pour constituer une grille d'évaluation des projets pouvant bénéficier des subventions du Fonds. Ces éléments sont à la fois liés à : la nature du programme ; l'ampleur du déficit en habitat; le niveau de pauvreté multidimensionnelle ; la convergence avec les autres programmes de l'Etat; le niveau de développement humain de la région et le niveau de développement économique de la région.

Ces critères d'appui pour attribuer la subvention du FSHIU sont susceptibles de créer un certain équilibre dans la répartition des financements et de cibler les différents territoires par les actions d'amélioration de la situation de l'habitat. En fait, chaque élément est doté d'un coefficient qui permet de calculer un score pour attribuer une subvention pour le projet (tableau 5).

TABLE V. CRITÈRES DE RÉPARTITION DES SUBVENTIONS FSHIU

\begin{tabular}{|c|c|c|}
\hline \multicolumn{2}{|r|}{ Critères } & Notes \\
\hline \multirow{5}{*}{$\begin{array}{l}\text { Nature du } \\
\text { projet }\end{array}$} & VSB & 8 pts \\
\hline & MANU & 4 pts \\
\hline & PV & 4 pts \\
\hline & HMR & 4 pts \\
\hline & Provinces Sud & 2 pts \\
\hline \multicolumn{2}{|c|}{ Ampleur du déficit en habitat } & $\begin{array}{l}\text { Plus de } 10 \%: 8 \text { pts de } 5-10 \%: 4 \text { pts moins de } 5 \%: \\
\qquad 2 \text { pts }\end{array}$ \\
\hline \multicolumn{2}{|c|}{ Niveau pauvreté multidimensionnelle } & $\begin{array}{l}\text { Taux de pauvreté }>10 \%: 8 \text { pts, de } 5 \text { à } 10 \%: 4 \text { pts } \\
\text { moins de } 5 \%: 2 \text { pts }\end{array}$ \\
\hline \multicolumn{2}{|c|}{$\begin{array}{c}\text { La convergence avec les autres } \\
\text { programmes de l'Etat }\end{array}$} & $\begin{array}{l}\text { Existence d'autres programmes sur le même site } \\
\text { d'intervention : } 4 \text { pts, non : } 0 \text { pts }\end{array}$ \\
\hline \multicolumn{2}{|c|}{$\begin{array}{l}\text { Niveau de développement humain de } \\
\text { la région }\end{array}$} & $\begin{array}{c}\text { Coefficient de développement humain de la région }> \\
\text { à la moyenne nationale : } 8 \mathrm{pts} \text {, non : } 0 \text { pts }\end{array}$ \\
\hline \multicolumn{2}{|c|}{$\begin{array}{l}\text { Niveau de développement économique } \\
\text { de la région }\end{array}$} & $\begin{array}{c}\text { PIB/tête de la région }>\text { la moyenne nationale }: 0 \text { pts, } \\
\text { non }: 8 \text { pts }\end{array}$ \\
\hline
\end{tabular}

À titre d'exemple, la comparaison entre 10 dossiers (demandes) d'intervention va se faire sur la base des notes attribuées à travers la grille ci-dessus. Plus la note est élevée plus le demande est prioritaire. 
Effina et al.: Détermination d'un nouveau système de critères de transfert de l'Etat vers les collectivités territoriales...

TABLE VI. SiMUlation DES NOTES DE 10 PROJETS DE FINANCEMENT PAR LE FSUIU

\begin{tabular}{|c|c|c|c|c|c|c|c|}
\hline & $\begin{array}{c}\text { Nature } \\
\text { projet }\end{array}$ & $\begin{array}{l}\text { Ampleur du } \\
\text { déficit en } \\
\text { habitat }\end{array}$ & $\begin{array}{c}\text { Niveau } \\
\text { pauvreté } \\
\text { multidimensio } \\
\text { nnelle }\end{array}$ & $\begin{array}{c}\text { La convergence } \\
\text { avec les autres } \\
\text { programmes de } \\
\text { l'Etat }\end{array}$ & $\begin{array}{c}\text { Niveau de } \\
\text { développe } \\
\text { ment } \\
\text { humain }\end{array}$ & $\begin{array}{c}\text { Niveau de } \\
\text { développeme } \\
\text { nt } \\
\text { économique }\end{array}$ & Total \\
\hline D1 & VSB & 8 & 8 & 0 & 0 & 4 & 20 \\
\hline D2 & VSB & 8 & 8 & 4 & 4 & 4 & 28 \\
\hline D3 & MANU & 4 & 4 & 0 & 0 & 0 & 8 \\
\hline D4 & PV & 4 & 0 & 0 & 0 & 0 & 4 \\
\hline D5 & PS & 2 & 0 & 4 & 4 & 4 & 14 \\
\hline D6 & PS & 2 & 0 & 4 & 4 & 4 & 14 \\
\hline D7 & HMR & 5 & 0 & 4 & 4 & 4 & 17 \\
\hline D8 & VSB & 8 & 4 & 0 & 0 & 0 & 12 \\
\hline D9 & MANU & 4 & 4 & 4 & 4 & 0 & 16 \\
\hline D10 & MANU & 4 & 8 & 4 & 4 & 0 & 20 \\
\hline
\end{tabular}

\section{USING THE TEMPLATE VUE D'ENSEMBLE DU NOUVEAU SYSTÈME DE CRITÈRES DU TRANSFERT DE L'ETAT VERS LES COLLECTIVITÉS}

Le nouveau système de critères encadrant les transferts financiers de l'Etat vers les collectivités territoriales s'inspire du cadre référentiel de la solidarité territoriale. Il est en conformité avec les principes fondamentaux et s'agit d'une déclinaison des orientations stratégiques de la solidarité territoriale et le principe de l'équité territoriale.

\section{A. Situation de la péréquation au Maroc et en France}

Au cours de ces dernières années, à l'instar des pays développés, la péréquation (art. 72.2 de la Constitution française) en France enregistre une émergence importante. Cela est dû aux spécificités du modèle de solidarité territoriale de ce pays. Il s'agit d'un modèle totalement différent du cas marocain. En effet, le Budget des collectivités en France représente 51\% du budget général de l'État contre seulement 9\% pour le Maroc. Cela dénote de l'état d'avancement du processus de décentralisation au Maroc en comparaison en France et montre également le rôle que jouent effectivement les collectivités au sein du développement local du pays (tableau 7).

TABLE VII. COMPARATIF DES FINANCES LOCALES DU MAROC ET DE LA FranCE

Cas français

Cas marocain

\begin{tabular}{c|r|r|r|r}
\cline { 2 - 5 } & En MM€ & \% & En MMDH & \% \\
\hline BGE de l'Etat & 385.9 & & 398.3 & \\
\hline Transfert au CL & 50.3 & $\mathbf{1 3 \%}$ & 24.2 & $\mathbf{6 \%}$ \\
\hline Budget CL & 197 & $\mathbf{5 1 \%}$ & 37.2 & $\mathbf{9 \%}$ \\
\hline Recettes de la fiscalité locale & 131 & $\mathbf{6 7 \%}$ & 7.1 & $\mathbf{1 9 \%}$ \\
\hline Population : & 66.6 & & 35.3 & \\
\hline Ratio BGE/Pop. (1) & 5794.3 & & 11289.1 & \\
\hline Ratio B-CL/Pop. (2) & 2958.0 & & 1053.1 & \\
\hline Rapport (2)/(1) & $\mathbf{5 1 \%}$ & $\mathbf{9 \%}$ & \\
\hline \multicolumn{7}{|c|}{ Source : Exercice 2016, $M E F$}
\end{tabular}


L'importance des collectivités territoriales est constatée à travers le budget transféré par l'État à ces dernières. Ce budget représente 13\% du Budget Général de l'Etat (BGE), contre seulement 6\% au Maroc. En plus, les collectivités en France disposent d'une fiscalité locale variée permettant de générer 67\% du Budget global des collectivités, contre seulement 19\% au Maroc. Le ratio du Budget de l'État par tête en comparaison à celui des collectivités territoriales par tête est de 51\% en France, contre seulement 9\% au Maroc (Figure 3).

La France distingue les ressources qui sont transférées ou produites directement par les collectivités en relation avec leur rôle règlementaire, d'autres ressources qui concernent la solidarité territoriale (péréquation) visant la réduction des disparités territoriales, mais à travers les collectivités directement. Les ressources dédiées à la péréquation en France représentent 10.8 MM $€$ en 2016, soit 5.5\% du budget global des collectivités. Près de $70.4 \%$ de ces ressources sont dédiées à la péréquation verticale et $29.6 \%$ à la péréquation horizontale.

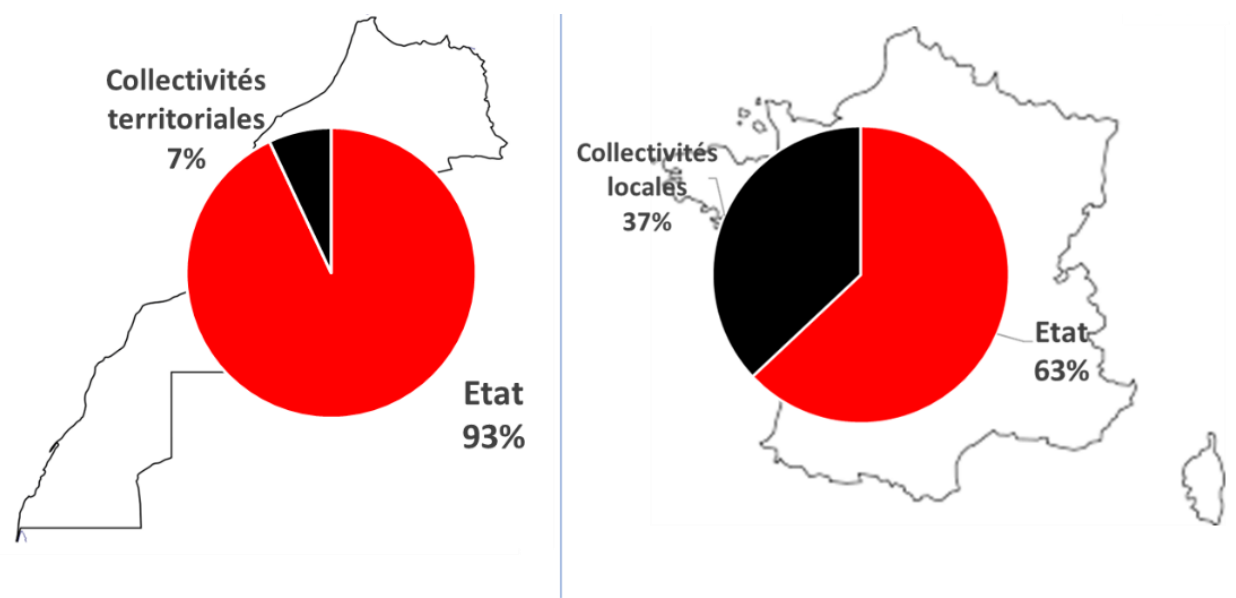

Figure 3. Répartition du budget général de la nation

Au Maroc, la péréquation territoriale, au sens français, se fait à travers le fonds de solidarité interrégionale (mise en place depuis 2018). En principe, les ressources de ce fonds sont transférées vers les régions, selon un certain nombre de critères techniques et socio-économiques. Toutefois, il y a lieu de mentionner que la solidarité territoriale se fait à travers plusieurs autres leviers. Mais, à la différence de la France, les ressources financières de ces instruments ne passent pas à travers les collectivités. En général, ces fonds sont gérés par les administrations centrales et leurs ressources ne sont pas réparties selon des critères connus à l'avance. C'est le cas notamment du fonds de solidarité de l'habitat et d'intégration urbaine (FSHIU).

Ce fonds, géré par le département de l'habitat et de la politique de la ville, créée depuis 2002, est doté d'un budget de 2 milliards de $\mathrm{DH}$. Les ressources de ce fonds proviennent principalement de la taxe spéciale sur le ciment et la taxe sur le fer à béton. L'analyse de l'historique de la répartition des ressources de ce fonds montre une certaine concentration des interventions dans certaines régions par rapport d'autres. Ce fonds ne dispose pas à aujourd'hui de critères de réparation de ces ressources sachant que les domaines de son intervention sont règlementés. En plus, ce fonds a joué un rôle important dans la réduction de l'habitat insalubre au niveau d'un grand nombre de communes. Dans ce cadre, l'on peut faire ressortir les constats stratégiques suivants :

- Le processus de décentralisation est long au Maroc. Quelles que soient les compétences et les attributions transférées aux collectivités, elles ne peuvent avoir d'effet réel que si elles sont accompagnées par les ressources financières nécessaires et des moyens permettant une gouvernance transparente et efficiente ;

- Le transfert des compétences aux régions doit être accompagné par le transfert des ressources financières et humaines ; 
- Les collectivités territoriales ont un rôle marginal dans le développement. En revanche, l'État reste l'acteur principal dans le développement territorial à travers un budget qui ne prend pas en considération les disparités territoriales, selon une grille de critères connue à l'avance ;

- La fiscalité locale en vigueur ne permet pas aux collectivités de disposer de suffisamment de ressources pour faire face aux déficits locaux et accélérer le processus de développement sur leurs territoires ;

- Malgré leur faiblesse, une partie des ressources de la fiscalité locale est gérée par l'État directement pour le compte des collectivités, ce qui affaiblit les possibilités de priorisation des projets par les élus locaux ;

- Le modèle de solidarité territoriale national est basé principalement sur des leviers de financement dont la gestion est toujours centralisée au niveau des administrations centrales des différents départements ministériels. Les ressources consacrées à la solidarité territoriale ne passent pas encore à travers les collectivités. En plus, on enregistre l'absence de critères de répartition des ressources de ces leviers de financement de solidarité territoriale à exception du FSI récemment créée.

En matière d'intervention dans le développement local et la réduction des disparités territoriales, la diversité des instruments existants peut représenter une force et un atout s'il est possible d'introduire les ajustements nécessaires visant l'amélioration du modèle de solidarité territoriale présenté ci-haut.

\section{B. Introduction des leviers de solidarité infrarégionale}

Il est suggéré de procéder à la mise en place d'un système de solidarité infrarégionale au niveau des régions relativement. C'est le cas des régions de Casablanca-Settat, Rabat-Salé-Kénitra et Tanger-Tétouan-Al Hoceima. Ce système consiste à la mise en place des fonds de solidarité à l'échelle de ces trois régions. Le but de ces fonds est de redistribuer une partie des ressources fiscales propres des collectivités riches vers les communes pauvres des mêmes régions. Par la suite, cette expérience peut être élargie à l'ensemble des régions.

En effet, le système propose une nouvelle démarche pour définir les budgets des régions et communes en tenant compte de la population et du niveau de développement social de chaque territoire. Il repose sur la prise en compte des déficits sociaux les plus importants pour répartir équitablement le budget entre les collectivités territoriales (tableau 8).

TABLE VIII. LES 7 DÉFICITS SOCIAUX DE BASE

\begin{tabular}{|c|c|c|c|}
\hline Type de déficit & Urbain & Rural & Ensemble \\
\hline 1. L'accès à l'électricité & $\mathrm{X}$ & $\mathrm{X}$ & $\mathrm{X}$ \\
\hline 2. L'accès à l'eau potable & $\mathrm{X}$ & $\mathrm{X}$ & $\mathrm{X}$ \\
\hline 3. L'accès à l'assainissement & $\mathrm{X}$ & $\mathrm{X}$ & $\mathrm{X}$ \\
\hline 4. La scolarisation de base & $\mathrm{X}$ & $\mathrm{X}$ & $\mathrm{X}$ \\
\hline 5. Les ménages encore dans des bidonvilles & $\mathrm{X}$ & & $\mathrm{X}$ \\
\hline 6. Déficit en routes rurales & & $\mathrm{X}$ & $\mathrm{X}$ \\
\hline $\begin{array}{c}\text { 7. Déficit en couverture sanitaire mesuré par le nombre de médecins pour } \\
\text { 10000 habitants }\end{array}$ & $\mathrm{X}$ & $\mathrm{X}$ & $\mathrm{X}$ \\
\hline
\end{tabular}

Source : Réalisé par nos soins

Ce nouveau système traduit le mécanisme central de l'opérationnalisation de la solidarité territoriale qui est la péréquation verticale. La matrice stratégique suivante retrace d'une façon synthétique la nouvelle architecture du système de répartition des transferts financiers de l'Etat vers les collectivités. 
Int. J. Fin. Acc. Eco. Man. Aud. 3, No. 3, 35-53 (May-2021)

TABLE IX. BUDGET DES CT PAR TÊTE ET SELON LES RÉGIONS EN 2018

\begin{tabular}{|c|c|c|c|c|c|c|c|c|}
\hline Régions & $\begin{array}{c}\text { Budget CT } \\
2018(*)\end{array}$ & Part \% & $\begin{array}{l}\text { Part \% } \\
\text { pop. }\end{array}$ & $\begin{array}{c}\text { Budget } \\
\text { CT/tête (DH) }\end{array}$ & $\begin{array}{l}\text { Superficie } \\
\left(\mathbf{k m}^{2}\right)\end{array}$ & $\begin{array}{l}\text { Part \% } \\
\text { Surface }\end{array}$ & IDSR & $\begin{array}{l}\text { Budget } \\
\mathrm{CT} / \mathrm{km}^{2}\end{array}$ \\
\hline Tanger-Tétouan-Al Hoceima & 4031 & $9,6 \%$ & $10,6 \%$ & 1082,1 & 17262 & $2 \%$ & 90,6 & 233519 \\
\hline Oriental & 3782 & $9,0 \%$ & $6,8 \%$ & 1574,3 & 90127 & $13 \%$ & 109,1 & 41963 \\
\hline Fès-Meknès & 4291 & $10,2 \%$ & $12,3 \%$ & 986,9 & 14395 & $2 \%$ & 84,1 & 298090 \\
\hline Rabat-Salé-Kénitra & 5602 & $13,3 \%$ & $13,5 \%$ & 1174,6 & 18194 & $3 \%$ & 74,6 & 307904 \\
\hline Béni Mellal-Khénifra & 2741 & $6,5 \%$ & $7,3 \%$ & 1061,7 & 28088 & $4 \%$ & 110,4 & 97586 \\
\hline Casablanca-Settat & 8507 & $20,3 \%$ & $20,5 \%$ & 1178,6 & 20166 & $3 \%$ & 65,7 & 421849 \\
\hline Marrakech-Safi & 4671 & $11,1 \%$ & $13,3 \%$ & 996,4 & 39167 & $6 \%$ & 102,4 & 119259 \\
\hline Draa-Tafilalet & 1908 & $4,5 \%$ & $4,8 \%$ & 1139,9 & 88836 & $12 \%$ & 94,1 & 21478 \\
\hline Souss-Massa & 2949 & $7,0 \%$ & $8,0 \%$ & 1046,8 & 51642 & $7 \%$ & 84,7 & 57105 \\
\hline Guelmim-Oued Noun & 1032 & $2,5 \%$ & $1,3 \%$ & 2335,9 & 52577 & $7 \%$ & 91,3 & 19629 \\
\hline Laayoune-Sakia El Hamra & 1756 & $4,2 \%$ & $1,1 \%$ & 4515,3 & 140018 & $20 \%$ & 54,5 & 12541 \\
\hline Dakhla-Oued Ed Dahab & 710 & $1,7 \%$ & $0,5 \%$ & 4296,5 & 142865 & $20 \%$ & 37,5 & 4970 \\
\hline National & 41980 & $100 \%$ & $100 \%$ & 1192 & 703337 & $100 \%$ & 85,85 & 53956 \\
\hline
\end{tabular}

(*) en millions de dirham ; Source : Réalisé par nos soins (HCP, MEF)

L'analyse de la répartition régionale du budget des collectivités montre des disparités importantes qui ne tiennent pas compte de la dimension démographique. En effet, les données relatives à la répartition régionale du budget des collectivités de 2018, montrent que la région du CS s'accapare près $20.5 \%$ de ce budget, suivi de la région RSK et la région de MS. La comparaison de cette réparation régionale du budget globale des collectivités révèle un gap important par rapport à la répartition de la population entre les régions. La région de FM à titre d'exemple qui représente un poids démographique de $12.3 \%$ ne bénéficie que de $10.2 \%$ du budget global des collectivités. Aussi la région MS qui représente $13.3 \%$ de la population ne bénéficie que de $11.1 \%$ du budget global alloué aux collectivités (tableau 9).

TABLE X. BUDGET DES COLLECTIVITÉS RELATIF DES RÉGIONS RAPPORTÉ AU BUDGET CT MOYEN EN COMPARAISON AVEC L'IDSR RELATIF EN 2018

\begin{tabular}{|c|c|c|c|c|c|c|}
\hline Régions & $\begin{array}{c}\text { Budget CT/ } \\
\text { tête (DH) X1 }\end{array}$ & IDSR Y1 & $\begin{array}{l}\text { Rapport } \\
\text { (X1/X2) }\end{array}$ & Situation/Budget & $\begin{array}{l}\text { Rapport } \\
\text { (Y1/Y2) }\end{array}$ & Situation/IDSR \\
\hline Tanger-Tétouan-Al Hoceima & 1082,1 & 90,6 & 0,91 & Cas normal & 1,05 & Situation normale \\
\hline Oriental & 1574,3 & 109,1 & 1,32 & Cas favorable & 1,27 & Situation relativement difficile \\
\hline Fès-Meknès & 986,9 & 84,1 & 0,83 & Cas défavorable & 0,98 & Situation normale \\
\hline Rabat-Salé-Kénitra & 1174,6 & 74,6 & 0,99 & Cas normal & 0,87 & Situation favorable \\
\hline Béni Mellal-Khénifra & 1061,7 & 110,4 & 0,89 & Cas normal & 1,29 & Situation relativement difficile \\
\hline Casablanca-Settat & 1178,6 & 65,7 & 0,99 & Cas normal & 0,77 & Situation favorable \\
\hline Marrakech-Safi & 996,4 & 102,4 & 0,84 & Cas défavorable & 1,19 & Situation relativement difficile \\
\hline Draa-Tafilalet & 1139,9 & 94,1 & 0,96 & Cas normal & 1,1 & Situation normale \\
\hline Souss-Massa & 1046,8 & 84,7 & 0,88 & Cas défavorable & 0,99 & Situation normale \\
\hline Guelmim-Oued Noun & 2335,9 & 91,3 & 1,96 & Cas très favorable & 1,06 & Situation normale \\
\hline Laayoune-Sakia El Hamra & 4515,3 & 54,5 & 3,79 & Cas très favorable & 0,63 & Situation très favorable \\
\hline Dakhla-Oued Ed Dahab & 4296,5 & 37,5 & 3,60 & Cas très favorable & 0,44 & Situation très favorable \\
\hline Moyenne nationale & 1192 & 85,85 & 1 & & 1 & \\
\hline Paramètres & $\mathrm{X} 2$ & Y2 & & & & \\
\hline
\end{tabular}

Le ratio budget des collectivités par tête des régions révèle un niveau élevé de disparité. Ce ratio varié de 986.9 dirhams pour la région de FM à 4515.3 dirhams pour la région LSH. 
La comparaison de ce ratio à l'IDSR (Indice des disparités sociales des régions) montre aussi que les budgets alloués aux collectivités ne reflètent pas le niveau important des déficits sociaux dans ces régions. L'approfondissement de l'analyse à travers la comparaison du ratio budget collectivités par tête et l'IDSR montre que :

- Deux régions se trouvent dans une situation très favorable (DOD et LSH), elles bénéficient d'un budget/tête supérieur à la moyenne nationale et un déficit cumulé inférieur à la moyenne nationale ;

- Deux régions se trouvent dans une situation favorable (RSK, CS). Elles bénéficient d'un budget/tête légèrement supérieur à la moyenne nationale et un déficit cumulé sensiblement inférieur à la moyenne nationale ;

- Cinq régions se trouvent dans une situation normale (GON, SM, DT, FM et TTH). Les budget/tête de ces régions sont poches de la moyenne nationale et leurs déficits cumulés et aussi proches de la moyenne nationale ;

- Trois régions se trouvent dans une situation relativement difficile, il s'agit des régions de l'Oriental, Beni Mellal-Khénifra et Marrakech-Safi.

La normalisation de la situation des régions passe nécessairement par l'adoption d'un nouveau système de critères de distribution du budget global des collectivités capable de tenir compte du poids démographique des régions et leur stock en différents déficits sociaux.

\section{REFORME PARAMETRIQUE DE LA REPARTITION BUDGETAIRE ET REPARTITION DE L'INVESTISSEMENT PUBLIC}

Le modèle proposé des transferts de l'Etat vers les collectivités territoriales se présente à deux niveaux : une répartition sous forme de dotations budgétaires pour les régions et les communes et une répartition des investissements publics entre les régions.

\section{A. Définition du budget des collectivités territoriales}

Le diagnostic a démontré que le budget actuel des collectivités est la résultante des dotations financières allouées par l'Etat et des ressources propres issues de la fiscalité locale instituée pour les collectivités. La répartition des dotations de l'Etat prend en considération les critères de la population, de la superficie et des ressources propres de chaque collectivité. Toutefois, le modèle proposé de définition des budgets des collectivités territoriales repose sur des formules précises en se référant à des critères objectifs et rationnels. Quatre critères essentiels constituent le socle du modèle de définition des budgets des collectivités. Il s'agit de :

- Une distinction claire et nette entre les communes à caractère urbain et à caractère rural ;

- Une place centrale du citoyen dans la définition du budget ;

- Une prise en considération du niveau de développement social des territoires ;

- Une quête de l'équité entre les territoires et la lutte contre l'enrichissement des territoires au détriment des autres.

Les formules qui encadrent la définition des budgets des collectivités se présentent pour chaque catégorie des collectivités territoriales, comme indiqué ci-dessous : 


\section{i. Réforme paramétrique de la répartition budgétaire entre les régions}

Le budget de la région (BR) doit prendre en considération :

1. la taille de la population

2. le niveau de développement social du territoire de la région

Avec :

$$
B R=\operatorname{pop}(R) *\left(\frac{B T R}{t \hat{t} t e}\right) *(1+a)
$$

$\mathrm{BR} /$ tête : Budget de la Région (BR) divisé par nombre d'habitant de la région

BTR : Budget total des régions (c'est la somme des budgets de 12 régions)

BTR/tête : Budget Total des 12 Régions divisé par le total de population nationale

$\operatorname{Pop}(\mathrm{R})$ : Population de la région

a : Indice de réduction des disparités régionales. Il varie de -1 à 1 . En principe, lorsque la région est trop déficitaire l'indice tend vers +1 . Lorsque la situation est normale il tend vers 0 et lorsque la région est bien favorable il tend vert -1 .

Cet indice est calculé à partir des 7 principaux indicateurs des déficits sociaux enregistrés au niveau de la région.

TABLE XI. TABLEAU DES VALEURS DES VARIABLES QUI COMPOSENT L'INDICE PAR TRANCHES DE DÉFICIT

\begin{tabular}{|c|c|c|c|c|c|}
\hline & \multicolumn{5}{|c|}{ Ecart à la moyenne nationale (MN) } \\
\hline & $\begin{array}{c}\text { moins de } \\
-25 \%\end{array}$ & $\begin{array}{c}\text { de }-10 \% \text { à } \\
-25 \%\end{array}$ & $\begin{array}{c}-10 \% \text { à } \\
+10 \%\end{array}$ & $\begin{array}{c}+10 \% \text { à } \\
25 \%\end{array}$ & $\begin{array}{c}\text { plus de } \\
25 \%\end{array}$ \\
\hline 1. Taux d'accès à l'électricité & -2 & 1 & 0 & 1 & 2 \\
\hline 2. Taux d'accès à l'eau potable & -2 & 1 & 0 & 1 & 2 \\
\hline 3. Taux d'accès à l'assainissement & -2 & 1 & 0 & 1 & 2 \\
\hline 4. Taux de scolarisation & -2 & 1 & 0 & 1 & 2 \\
\hline 5. Taux des ménages en bidonvilles & -2 & 1 & 0 & 1 & 2 \\
\hline $\begin{array}{l}\text { 6. Taux d'enclavement/Déficit en routes } \\
\text { rurales }\end{array}$ & -2 & 1 & 0 & 1 & 2 \\
\hline $\begin{array}{l}\text { 7. Nombre de médecins pour } 10000 \\
\text { habitants }\end{array}$ & -2 & 1 & 0 & 1 & 2 \\
\hline Total $(\mathrm{X})$ & -14 & 7 & 0 & 7 & 14 \\
\hline Indice $(\mathrm{X} / 14)$ & 1 & 0.5 & 0 & -0.5 & -1 \\
\hline
\end{tabular}

La détermination du budget de la région sous cette formule permet de prendre en considération l'importance de la population régionale et le niveau des déficits sociaux enregistrés au sein des territoires de la région. Les paramètres contenus dans la formule proposée sont à la fois objectifs et mesurés suivant des méthodes statistiques rigoureuses. Ainsi, cette formule permet de réduire davantage les écarts entre les 12 régions du pays en termes de dotations financières. En outre, elle offre la possibilité de cibler le citoyen quel que soit son territoire de résidence. 


\section{Simulation de la valeur de l'indice pour le cas de la région Souss-Massa}

A travers cette simulation, le but est de vérifier l'effet de la prise en considération de l'écart en termes des déficits qui existent entre la moyenne nationale et le niveau régional, dans la détermination du budget des régions. Pour le cas de la région Souss-Massa, les chiffres se présentent comme suit :

TABLE XII. SimUlation D’ÉCARTS POUR LA RÉGION SOUSS MASSA

\begin{tabular}{|c|c|c|c|c|}
\hline & Moyenne. Nat. & Région SM & Ratio : SM/Nat. & Note \\
\hline 1. Taux d'accès à l'électricité & 95 & 96 & $1 \%$ & 0 \\
\hline 2. Taux d'accès à l'eau potable & 96 & 93 & $-3 \%$ & 0 \\
\hline 3. Taux d'accès à l'assainissement & 85 & 82 & $-4 \%$ & 0 \\
\hline 4. Taux de scolarisation & 95 & 97 & $2 \%$ & 0 \\
\hline 5. Taux des ménages en bidonvilles & 4.4 & 5.1 & $16 \%$ & 1 \\
\hline 6. Taux d'enclavement/Déficit en routes rurales & 3.1 & 4.1 & $32 \%$ & 2 \\
\hline 7. Nombre de médecins pour 10000 habitants & 7.3 & 6.5 & $-11 \%$ & -1 \\
\hline Total & & & & 2 \\
\hline Indice & & & & $14.3 \%$ \\
\hline
\end{tabular}

Source : Réalisé par nos soins

Sur la base de cette simulation, le budget de la région Souss-Massa doit être augmenté de 14,3\%. Concernant la correction du budget de la région en fonction de la taille de la population, il y a lieu de montrer l'écart en terme BR/tête des régions selon les données de 2018.

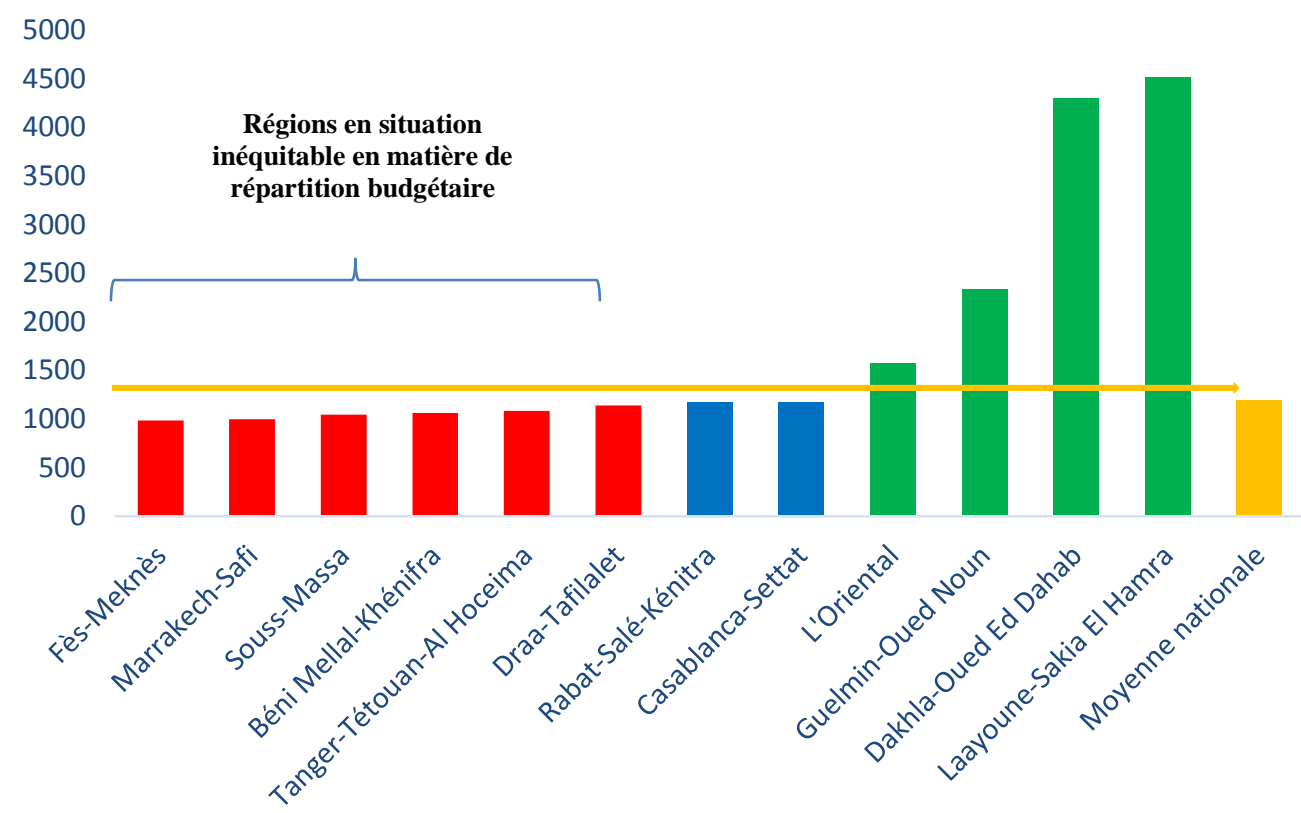

Source : (HCP, TGR), 2018

Figure 4. BR/tête selon les régions du Maroc 2018 


\section{Simulation de la valeur budgétaire de la région Fès-Meknès}

Dans cette simulation, le but est de vérifier l'effet de la prise en considération de la population dans la détermination du budget des régions. Pour le cas de la région FM, les chiffres se présentent comme suit :

TABLE XIII. BUDGET DE LA RÉGION FM SELON LE NOUVEAU MODÈLE

\begin{tabular}{ccc}
\hline Budget total régions/tête national & 1191.95 & Dhs \\
\hline Budget région FM/tête & 986.90 & Dhs \\
\hline Ecart & $-17.2 \%$ & \\
\hline Redressement \% & $17.2 \%$ & \\
\hline Ancien budget & 4291.00 & Millions de dhs \\
\hline Population & 4347958.00 & \\
\hline Nouveau budget & 5029.05 & Millions de dhs \\
\hline
\end{tabular}

Source : Réalisé par nos soins

Selon les calculs ci-dessus, le budget de la région FM doit être augmenté de 17.2\%. Il doit passer de 4.29 MMDH à 5.03 MMDH.

\section{ii. Réforme paramétrique de la répartition budgétaire entre communes}

En principe, la répartition budgétaire des communes doit obéir à deux règles :

1. La population de chaque commune ;

2. Le niveau des déficits sociaux sur les territoires des communes.

Le budget d'une commune à caractère urbain est défini à travers la formule suivante :

Avec :

$$
B R=\operatorname{pop}(M) *\left(\frac{B C T(M)}{t \hat{t} t e}\right) *(1+a)
$$

$B C(M)$ : Budget de la commune en milieu/rural

$B C T(M) / t e ̂ t e$ : Budget total des Communes (selon le milieu) par nombre de population de la région par milieu (urbain/rural)

Pop $(M)$ : population de la commune

$a$ : est l'indice de réduction des disparités intra région. Il varie de -1 à 1 . En principe lorsque la commune est trop déficitaire l'indice tend vers +1 . Lorsque la situation est normale il tend vers 0 et lorsque la commune est bien favorable il tend vert -1 .

Cet indice est calculé à partir des 7 principaux indicateurs des déficits sociaux enregistrés au niveau de la commune.

Le calcul du budget des communes sous cette formule permet de prendre en compte les spécificités de chaque commune par milieu. Elle permet aussi de cibler la population et répondre à ses besoins notamment en matière de services publics de base garantis par les communes. En effet, les différences entre les communes seront réduites en termes du budget. Ce dernier sera fonction de la population et de l'ampleur des déficits enregistrés au sein des communes concernées à l'intérieur de chaque région. 


\section{B. Répartition régionale de l'investissement public}

L'investissement public a toujours été un outil important pour la mise à niveau des territoires et la lutte contre les inégalités territoriales. Cet investissement, comme il a été souligné dans le diagnostic stratégique, connait une répartition inégale entre les régions. Cette situation est génératrice d'inégalités. La solidarité territoriale exige une répartition équitable des ressources financières allouées à l'investissement notamment sa composante sociale. Cette répartition doit prendre en considération les besoins des territoires et leur niveau de développement. Elle doit aussi regrouper les efforts d'investissement public en consolidant la part du Budget Général de l'Etat, des Etablissements et Entreprises Publiques et des Comptes Spéciaux de Trésor (CST).

Le modèle de répartition proposé repose sur le choix de la région comme échelle territoriale de convergence des politiques publiques. Il met ainsi la région en position centrale pour déterminer l'orientation des financements drainés en fonction de sa vision et de son plan de développement régional conformément au schéma régional d'aménagement du territoire (SRAT) et au plan de développement régional (PDR). Cette répartition permet également de cibler les territoires nécessiteux au niveau de chaque région et de booster l'action de développement territorial harmonieux. Elle est de nature à créer un équilibre entre les régions et entre les provinces, préfectures et communes de la région. C'est une réponse adaptée aux besoins de chaque territoire et une optimisation rationnelle de l'effort de l'investissement public.

Afin de rendre l'investissement public plus équitable entre les territoires, nous proposons un certain nombre de critères qui doivent être respectés dans la préparation l'investissement public, dans sa composante sociale, à l'échelle des régions :

1. Déficits sociaux cumulés : en principe l'investissement public doit s'orienter le plus vers les territoires qui enregistrent les déficits les plus élevés par rapport à la moyenne nationale. L'indice qui mesure l'intensité des déficits ci-après peut servir pour cartographier les territoires selon le degré de priorité d'intervention des politiques publiques.

TABLE XIV. CALCUl DE L'INDICE DE MESURE DES DÉFICITS SOCIAUX CUMUlÉS

\begin{tabular}{|c|c|c|c|c|c|}
\hline & \multicolumn{5}{|c|}{ Ecart à la moyenne nationale (MN) } \\
\hline & $\begin{array}{c}\text { moins } \\
\text { de }-25 \%\end{array}$ & $\begin{array}{c}\text { de }-10 \% \text { à } \\
-25 \%\end{array}$ & $\begin{array}{c}-10 \% \text { à } \\
+10 \%\end{array}$ & $\begin{array}{c}+10 \% \text { à } \\
25 \%\end{array}$ & $\begin{array}{c}\text { plus de } \\
25 \%\end{array}$ \\
\hline & $\begin{array}{c}\text { très } \\
\text { prioritaire }\end{array}$ & Prioritaire & normale & $\begin{array}{c}\text { moins } \\
\text { prioritaire }\end{array}$ & $\begin{array}{c}\text { Non } \\
\text { prioritaire }\end{array}$ \\
\hline 1. Taux d'accès à l'électricité & 2 & 1 & 0 & -1 & -2 \\
\hline 2. Taux d'accès à l'eau potable & 2 & 1 & 0 & -1 & -2 \\
\hline 3. Taux d'accès à l'assainissement & 2 & 1 & 0 & -1 & -2 \\
\hline 4. Taux de scolarisation & 2 & 1 & 0 & -1 & -2 \\
\hline 5. Taux des ménages en bidonvilles & 2 & 1 & 0 & -1 & -2 \\
\hline 6. Taux d'enclavement/Déficit en routes rurales & 2 & 1 & 0 & -1 & -2 \\
\hline 7. Nombre de médecins pour 10000 habitants & 2 & 1 & 0 & -1 & -2 \\
\hline $\operatorname{Total}(\mathrm{X})$ & 14 & 7 & 0 & -7 & -14 \\
\hline Indice $(\mathrm{X} / 14)$ & 1 & 0.5 & 0 & -0.5 & -1 \\
\hline
\end{tabular}


2. L'ancienneté des déficits sociaux : est un critère important dans la répartition des investissements publics et dans la priorisation des interventions de politiques publiques. Les données montrent que certains territoires enregistrent des déficits très anciens. Malgré cette situation, ces territoires n'arrivent pas à attirer l'attention des pouvoirs publics. Cette situation participe en général à de fortes immigrations de populations de ces territoires. L'indice ci-après peut servir pour cartographier les territoires selon le degré de priorité d'intervention des politiques publiques.

TABLE XV. CALCUL DE L’INDICE DE MESURE DE L’ANCIENNETÉ DES DÉFICITS

\begin{tabular}{|c|c|c|c|}
\hline & \multicolumn{3}{|c|}{ Ancienneté des déficits } \\
\hline & Plus de 20 ans & De 10 à 20 ans & Moins de 10 ans \\
\hline 1. Taux de chômage par région & 3 & 2 & 1 \\
\hline 2. Taux d'accès à l'électricité & 3 & 2 & 1 \\
\hline 3. Taux d'accès à l'eau potable & 3 & 2 & 1 \\
\hline 4. Taux d'accès à l'assainissement & 3 & 2 & 1 \\
\hline 5. Taux de scolarisation & 3 & 2 & 1 \\
\hline 6. Taux des ménages en bidonvilles & 3 & 2 & 1 \\
\hline 7. Taux d'enclavement/Déficit en routes rurales & 3 & 2 & 1 \\
\hline 8. Nombre de médecins pour 10000 habitants & 3 & 2 & 1 \\
\hline 9. Taux d'abandon scolaire & 3 & 2 & $\mathbf{7}$ \\
\hline
\end{tabular}

Source : Réalisé par nos soins

3. En plus du niveau global des déficits sociaux et de l'ancienneté de ces déficits, certains secteurs prioritaires peuvent représenter un critère à part de répartition de l'investissement public. Les déficits enregistrés au niveau des secteurs de l'enseignement, la santé, les routes et l'habitat peuvent représenter un critère important dans la répartition des investissements. Cela nécessite un système de pondération des 7 types de déficits précédemment cités.

TABLE XVI. CALCUL DE L'INDICE POUR LES SECTEURS PRIORITAIRES

\begin{tabular}{|c|c|c|}
\hline & \multicolumn{2}{|c|}{ Système de pondération } \\
\hline Domaines des déficits & variante 1 & variante 2 \\
\hline 1. Taux d'accès à l'électricité & $14 \%$ & $10 \%$ \\
\hline 2. Taux d'accès à l'eau potable & $14 \%$ & $10 \%$ \\
\hline 3. Taux d'accès à l'assainissement & $14 \%$ & $10 \%$ \\
\hline 4. Taux de scolarisation & $14 \%$ & $25 \%$ \\
\hline 5. Taux des ménages en bidonvilles & $14 \%$ & $10 \%$ \\
\hline 6. Taux d'enclavement/Déficit en routes rurales & $14 \%$ & $10 \%$ \\
\hline 7. Nombre de médecins pour 10000 habitants & $14 \%$ & $25 \%$ \\
\hline Total & $100 \%$ & $100 \%$ \\
\hline
\end{tabular}

Source : Réalisé par nos soins

4. Les données montrent que certains territoires spécifiques doivent être prioritaires dans les interventions publiques. C'est le cas des zones de montagne, les zones de frontières et les Oasis. Les indicateurs sociaux de ces zones sont très alarmants.

La répartition de l'investissement public entre les régions selon la formule proposée se réfère toujours des principes d'équité, de ciblage des citoyens et de réduction continue des disparités entre les territoires régionaux. 
En effet, cette formule garantit pour tous les territoires de recevoir un minimum de fonds pour réaliser les investissements nécessaires pour la lutte contre les inégalités territoriales notamment sociales. Elle permet de diversifier les sources de financement des régions et garantir leur pérennité.

Une fois la part des régions en investissement public est déterminée, sa répartition entre les provinces et les préfectures est laissée pour la Région. En fait, cette répartition infrarégionale est tributaire de la planification du développement au niveau des régions. Le SRAT en tant que référence de l'aménagement du territoire et base de détermination des espaces-projets permet de définir les territoires prioritaires à recevoir des investissements à même de mettre à niveau les espaces dépourvus. De plus, le PDR comme déclinaison opérationnelle de la volonté de développement, est l'occasion pour répartir la dotation en investissement entre les territoires et financer les différents projets de lutte contre les inégalités territoriales.

Ladite répartition doit être soumise à la contractualisation entre l'Etat et la Région et aussi à la coopération et le partenariat entre ces régions et les Etablissements et Entreprises Publiques (EEP). Ces pratiques de partenariat et de contractualisation, prévues par les différents textes législatifs et règlementaires en vigueur, permettront de concrétiser davantage ce mécanisme de répartition inter et infra régionale de l'investissement public. En plus, la déconcentration administrative donnant plus d'autonomie pour les services de l'Etat soutient cette tendance et permet de consolider la contractualisation entre l'Etat et les régions.

Par ailleurs, la nouvelle architecture budgétaire de l'Etat basée sur la décentralisation budgétaire permet de concrétiser cette répartition proposée. En fait, la régionalisation des crédits selon des programmes et des projets renforce davantage cette tendance de répartition et de solidarité régionale. Cependant, l'octroi de ressources financières aux Régions pour concrétiser leurs nouvelles compétences et responsabilités doit être accompagné par des mesures de planification budgétaire. Il s'agit alors d'insérer l'action de développement territorial dans une logique de continuité et de pluri annualité conformément au nouveau principe budgétaire de programmation pluriannuelle. Ceci est garant d'une meilleure utilisation des parts de l'investissement public allouées aux Régions pour lutter contre les inégalités territoriales.

\section{CONCLUSION}

La mise en place d'une nouvelle matrice de critères de transfert financier de l'Etat vers les collectivités est sûrement un pas nécessaire pour la réforme du système de solidarité territoriale. L'adoption de cette nouvelle architecture est exigée par la nouvelle configuration territoriale du Royaume basée sur la régionalisation avancée. Cette nouvelle organisation territoriale place la région au centre de l'action publique de lutte contre les inégalités territoriales. Cette action qui nécessite en premier lieu une mise à disposition des moyens nécessaires au profit des collectivités et par la suite une meilleure répartition de ces moyens au sein du territoire régional. L'adoption des critères prenant en compte la population, comme cible de l'action publique de développement, permet de satisfaire les besoins du citoyen quel que soit le territoire où il réside. C'est une concrétisation réelle des principes fondamentaux universels et constitutionnels ainsi qu'une garantie d'un ciblage efficace et efficient de toutes les catégories de population notamment les plus défavorisées.

En outre, la prise en considération des spécificités territoriales et de la nature du milieu de résidence des citoyens permet de répondre aux besoins réels de chaque territoire. Il est aussi d'une grande importance pour renforcer l'action publique destinée aux territoires sensibles en matière de développement socio-économique. La pondération des transferts par le niveau de développement socio-économique des territoires est de nature à prendre en compte d'une façon pratique la réalité des disparités territoriales existantes.

Ceci donnera l'occasion pour les territoires dépourvus d'être bien lotis en moyens financiers pour faire face aux retards enregistrés en matière de développement humain. De plus, la dépendance du budget d'une collectivité 
des autres est une garantie pour réduire les écarts entre les collectivités. Elle permet de maintenir un niveau d'équilibre entre les budgets des collectivités et de lutter contre les écarts importants entre communes et régions.

En outre, l'encadrement des ratios des budgets assure une juste répartition des moyens entre les collectivités. Le recours à une orientation des investissements publics vers les Régions et leur répartition selon les mêmes critères de population et de niveau du déficit socio-économique renforce davantage la position de la Région comme acteur de développement régional. Ainsi, le drainage des parts d'investissement public vers les territoires nécessiteux est tributaire d'une meilleure planification du développement territorial et d'une planification budgétaire pluriannuelle s'inscrivant dans la continuité de l'action publique.

Enfin, la concrétisation de la réforme proposée nécessite une coopération permanente entre les Régions, l'Etat et les Etablissements Publics. Cette collaboration sous forme de contractualisation ou de partenariat permet de renforcer la convergence des politiques publiques et la mutualisation des efforts pour plus de ciblage efficace des territoires. Ceci est garant de la performance du système de solidarité territoriale.

\section{REFERENCES}

[1] Cour des Comptes (2015), « Rapport annuel de la Cour des Comptes 2013 ».

[2] Constitution marocaine de 2011

[3] Décret n²-17-598 fixant les critères de distribution des recettes du fonds de solidarité interrégionale.

[4] HCP (2019), « Annuaires statistiques ».

[5] HCP (Octobre, 2018) « Le développement socio-économique-régional : Niveau des disparités, 2001-2017 ».

[6] HCP (2017), « Projections de la population des régions et des provinces 2014-2030».

[7] Loi organique 111-14 relative aux régions.

[8] Loi organique $\mathrm{n}^{\circ} 130-13$ relative à la loi de finances.

[9] Ministère de l'Aménagement du Territoire National, «L'Habitat en Chiffres », (2014-2019)

[10] Ministère des Finances (2019), « Rapport économique et financier ».

[11] Trésorerie Générale du Royaume, « Bulletin Mensuel de Statistiques des finances locales », 2016-2018. 